indicate that the latter gives an even more satisfactory result.

This new medium consists of a solution of Canada balsam in phenol (Phenol Liq. B.P.), prepared by heating the Canada balsam to drive off any water present, and adding phenol to the cooling mass until a satisfactory consistency is obtained. Larvæ are mounted direct from 95-100 per cent alcohol, the ventral surface of thorax and abdomen being first pierced in several places with a fine needle. Mounts clear rapidly and are sufficiently firm for use after several days in the sun.

A further refinement of the method, for use when perfectly cleared specimens are required for systematic research, etc., is to macerate specimens first in strong chloral hydrate solution (80 gm. in 20 c.c.) or 30 per cent acetic acid. Larvæ should be killed in hot water or other fixative and pierced with fine needles as above, and then placed in the chloral hydrate for twenty-four hours or more. They should then be washed in 90 per cent alcohol and given a short phenol bath prior to mounting in the phenol-balsam. As such specimens are very soft, the transfer to the slide should be made by pipette. Such mounts are perfectly transparent, being little more than larval skins, and are most useful where ventral hairs are to be examined. 'The method is, however, only applicable to fresh material; old specimens from alcohol fail to clear in chloral hydrate.

Bhatia's pine rosin has also been tested in phenol solution and gives a result equally as good as the balsam medium-sometimes a little better. But as the keeping qualities of the rosin have not yet been tested over a long period, I prefer to use balsam.

The above processes are presumably applicable to other small Arthropoda. A more detailed account is being prepared for publication elsewhere.

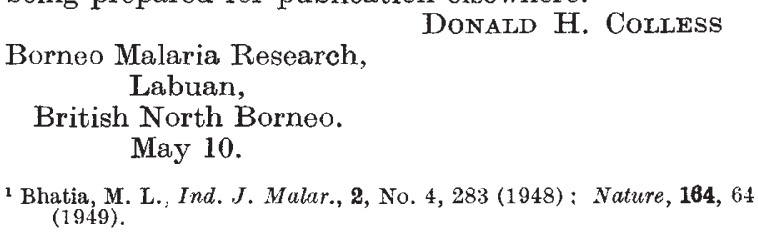

British North Borneo. May 10.

${ }^{1}$ Bhatia, M. L., Ind. J. Malar., 2, No. 4, 283 (1948) : Nature, 164, 64 (1949).

\section{Deposition of Air-borne Particles on Trap Surfaces}

STICKY traps in the form of vertical cylinders, and vertical or horizontal plates, are widely used to estimate the numbers of air-borne particles such as pollen, fungus spores, fungicides and insecticides. Although a knowledge of factors controlling deposition is a prerequisite for interpreting trap catches, the relation between the numbers deposited and their concentration in the air sweeping the surface has had scant attention. The problem has been studied here in connexion with epidemic crop diseases, and it has been shown that the size of the trap itself affects the catch to an extent greater than generally recognized.

When a plate or cylinder is placed with axis at right-angles to the wind, Sell ${ }^{1}$, working with droplets, has shown that as the size of the trap is increased an increasing proportion of the air stream is deflected by the streamlines and fails to strike the trap. The percentage of particles deposited from the column of air sweeping the surface gives a measure of the efficiency of the trap. The concentration of particles in the air can be measured directly with a poweroperated suction trap such as the Cascade impactor ${ }^{2}$ or a bubbler device. Efficiency can be shown to depend on particle size, wind speed and dimensions of trap. In a small wind tunnel operated at speeds between 1 and 10 metres per sec., the efficiencies of vertical cylinders of various diameters have been found to agree closely with the theoretical predictions of Glauert ${ }^{3}$. Table 1 gives illustrative values for Lycopodium clavatum spores (approximately $32 \mu$. in diameter) based on wind-tunnel and open air tests, and for spores of Lycoperdon giganteum (approximately $4 \mu$ in diameter) based on a small number of open-air tests only.

Table 1. Percentage Efficiency of Vertical Sticky Surfaceis (wind tunnel and open air tests)

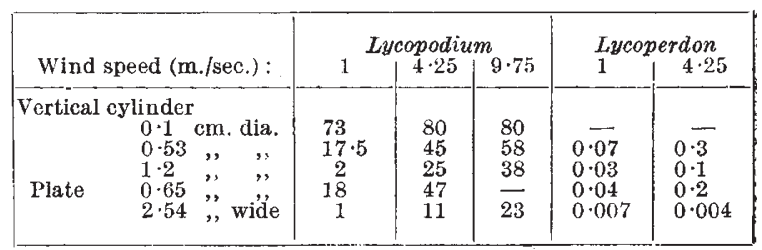

For horizontal plane surfaces, parallel to the air flow, such as the horizontal slides standard in pollen trapping, the principles of deposition are less clear. It is generally assumed that each particle-size is deposited by sedimentation at its characteristic terminal velocity 4 , the trap being 100 per cent efficient. However, wind tunnel tests, illustrated in Table 2 , show that anomalous values are obtained with horizontal glass slides. At wind speeds approaching 10 metres per sec. as many Lycopodium spores are deposited on the under surface of a horizontal slide as on the upper. Sedimentation under gravity must therefore play a minor part except at low wind speeds, and it is suggested that deposition is brought about largely by turbulent flow over the trap surface. The number deposited is, in fact, nearly proportional to the number of spores passing over the surface in a given time, irrespective of the amount of air they are diluted with, that is, irrespective of concentration.

Table 2. No. of Lycopodium spores deposited per min. from a cloud concentration of $16^{6}$ spores $/ \mathrm{m}^{3}$ on horizontal glass plate 3 in. $\times 1$ in. Wind speed (m./sec.) Upper surface in turbulent tunnel

$\begin{array}{ccccc}9 \cdot 2 & 5 \cdot 6 & 3 \cdot 2 & 1 \cdot 7 & 1 \cdot 1 \\ 140 & 78 & 12 & 15 & 43\end{array}$

Factors controlling deposition are evidently complex and not yet well understood. While a feathery grass stigma may catch pollen with high efficiency, many types of spore trap in current use are inefficient. Deposits on surface traps give direct information only on the amount of deposition on surfaces similar to themselves in dimensions and other properties. Although the intuitive belief that there is a general positive relation between deposition on a surface and concentration in the air is correct, the trap efficiency varies with conditions, sometimes widely enough to make a direct interpretation misleading. P. H. Gregory

Rothamsted Experimental Station, Harpenden, Herts.

$$
\text { May } 26 .
$$

${ }^{1}$ Sell, W., Forschungsheft, 347, 1 (1931).

${ }^{2}$ May, K. R., J. Sci. Instr., 22, 187 (1945).

Glauert, M., Aeronautical Research Committee, R. \& M. No. 2025 (London, H.M.S.O., 1940.)

- Cocke, E. C., J. Allergy, 9, 458 (1938). 